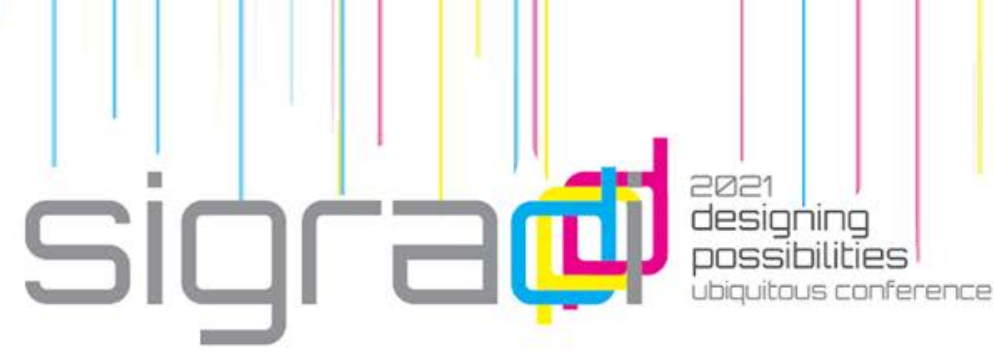

\title{
IDEAS: Interactive Database for Experimental Architecture and Spatial Practices
}

\author{
Raquel Lopes, Alexandra Paio \\ ISCTE-IUL Instituto Universitário de Lisboa, Portugal \\ rmgls@iscte-iul.pta \\ alexandra.paio@ iscte-iul.pt
}

\begin{abstract}
The interface of science and technology has become a fruitful transdisciplinary research field for spatial practices. To address this hybridization of space-related research, it is fundamental to ask what kind of practices are emerging within this context. To promote easy access and the dissemination of methodologies applied in innovative experimental spatial practices, the present study proposes the creation of a web-based Interactive Database for Experimental Architecture and Spatial Practices, IDEAS. Therefore, we will scope, observe, examine, and classify the digital cultural landscape in the 1960-2020 period. This paper describes the methodology applied to develop IDEAS.
\end{abstract}

Keywords: Spatial practices, Data gathering, Taxonomy, Architecture Wiki, Interactive Repository

\section{Introduction}

Architecture has always been defined by the prevailing means of production. Stonemasons once carved column capitals and modern architects harnessed the prefabricated components made possible by industrialisation (Young, 2019:8). Now multiplicity and plurality are celebrated, as flows, networks, and maps replace grids, structures, and history (Vidler, 2004:143).

The merging of art, science and technology presents new possibilities and mediums for design, creating emerging hybrid research fields and cultural expressions (Kak, 2007; Wilson, 2007; Gere, 2006). The place and impact of science and technology in the current cultural landscape - technoculture - has become a fruitful research field, focusing on the possibilities they present for transdisciplinary practices (Leopold et al, 2020; Leavy, 2017; Maldonado, 2012; Shaw, 2008; Cooper, 2002). Despite these important contributions, spatial practices are distant from the experimentations that could change how spaces are conceived and felt (Dave, 2005). In this paper the term "spatial practices" will be used to refer to transdisciplinary approaches, methodologies and projects that can contribute to conceive space and enhance public space in a 
digital culture (Melendez et al., 2021; Signore \& Riether, 2018; Leitão, 2012; Mueller, 2009; Liggett \& Perry, 1995). Lefebvre (1974) assigned spatial practices to the category of perceived space, the space of social relations and of experiences of daily life. Like Dodd (2020:11) we re-appropriate the term to mention forms of shaping practice "within and between the realms of art and architecture in more open-ended and socially engaged approach to the design of spaces".

To promote easy access and dissemination of innovative experimental spatial practices, the present study proposes the creation of a web-based Interactive Database for Experimental Architecture and Spatial practices (IDEAS). The power and importance of this interactive database is not to be underestimated. The inherent process of scoping, observing, examining, and classifying, is a prerequisite to develop a critical analysis about how innovative research and methodologies are being harnessed in the creation of experimental architecture. As Koolhaas (2019:60) states When our collective history is digital, the data centre is becoming one of our most significant cultural typologies.

This project is inspired by three key works: Charles Jencks's research and classification proposal presented in the books Architecture 2000: Methods and predictions (1971), the reviewed edition, 2000 and Beyond: Success in the art of prediction, and Peter Cook's challenge in Experimental Architecture (1970). The project proposes to combine these with the possibilities digital technologies offer to data gathering and classification. Besides that, we aim to create an operative tool to help practitioners exploring transdisciplinarity and boundary dissociation in spatial practices. As Carpo (2018:21) argues, information retrieval is increasingly (...) demoting all modern and traditional tools of story building and storytelling. This major anthropological upheaval challenges our ancestral dependence on shared master narratives.

IDEAS aims to create an operative tool for practitioners in preliminary stages of project development to research and understand how to expand their scope of action within the projects they are carrying. In this sense, the project will contribute to reconstrue the foundations of the discipline (...) in broader concepts that acknowledge an expanded field (Vidler, 2004:144).

The present paper is presenting the preliminary results of the ongoing research.

\section{Background}

Hybrid and transdisciplinary practices have changed cultural discourse and knowledge production. As Stalder argues:

Our daily lives, our culture and our politics are now shaped by the digital condition as large numbers of people involve themselves in contentious negotiations of meaning in ever more dimensions of life, from the trivial to the profound. (2018: 5) 
The accelerated datafication of society is challenging the collective forms of cooperation, requiring not only new critical thinking, but also new approaches for shaping our future data-driven society (Leopold et al, 2020). Transdisciplinarity and the merging of art, science, and technology in cultural practice, have been changing expanding the role of knowledge fields and their scope of action, namely architecture and urban spatial practices, towards more pratical and socially oriented practices (Bernstein, 2015; Stokols, 2006;).

Transdisciplinary architecture is an architecture of a hybrid nature, requiring other disciplines to target complex problems (Burry, 2012; Lawrence \& Després, 2004). Developments during the 1990s in architectural and urban theory showed a close dialogue with other disciplines. (...) where borders are blurring (Woiseth \& Nilsson, 2011:85).

Hybridity is a natural outcome when dealing with complexity and the expansion of a certain practice to a bigger field of action, requiring a layered solution to target all the problems that the project aims to solve. As Signore and Riether (2018:11) state "Hybrid public space refers to collectively inhabited urban space that is traversed by digital flows of data and images that enhance and alter the traditional interaction between the body and its physical, social and symbolic environment". To address the hybridization of knowledge production in space-related research and its impact on the contemporary cultural landscape is fundamental to question: how has the digital shift transformed the nature of the physical world? Consequently, how do architects acknowledge and interact with reality? (Fankhänel \& Lepik, 2020; Young, 2019; Carpo, 2017; Vidler, 2004; Zellner, 1999); What kind of spatial practices and spatial production are emerging within this context?

Today, discussions in architectural and urban spatial practices are shaped by many ideas. The conceptualizations and strategies are molded by influences emerging along the interface between science and technology. Buckminster Fuller coined design science revolution in 1961 through The World Design Science Decade, proposed to the International Union of Architects.

Ten years later, Charles Jencks's books Architecture 2000: Methods and Predictions (1971) and Beyond: Success in the Art of Prediction are organized a list of keywords to revise the discourses landscape on architectural practices. In the first book, Jencks intertwined architecture, art, politics, economy, in a cause-effect timeline, conceiving evolution with comprehensible streams of information (...) constructed from big data coming from everywhere (Jencks, 2015:129).

At the same time, Peter Cook's book Experimental Architecture (1970) is a manifesto that questions what architecture could be if it wasn't static, inspired by the technological automatization of the Second Machine Age: prefabrication, plug-in, clip-ons, pneumatic structures, and systems. Cook's radicalism relied on a critical exploration of the existent means.

Both authors and the mentioned books are an inspiration for this project: Jencks's predictions show the power of data abundance for classification and correlation; Cook invites creativity and a broader range of action for architecture with inter and transdisciplinarity. 
The notion of boundary dissociation and the combination of apparently disconnected knowledge fields to solve complex problems, was powered by the potential digital software was forecasting. Now, Big Data, neuroscience, digital fabrication, robotics, and artificial intelligence are inspiring multidisciplinary research to create spatial practices and experimentations that are concerned with the current philosophic ontology and environmental challenges. Regarding this, studying transdisciplinary approaches (Bernstein, 2015; Doucet \& Janssens, 2011; Burry, 2009; Stokols, 2006) enables the exploration of new possibilities and mediums that are emerging in the digital landscape.

\section{Methodology}

If it is easy to follow cultural practices from a zoomed-out point of view, zooming in, and study a project's methodology, is another story.

IDEAS is tracking, scoping, mapping and correlating processes and methodologies applied in different spatial practices to expose the diversity of knowledge fields involved, the used methodologies and technologies, the project stages, progresses and indents, the messy parts, and the key shifts for success.

Lev Manovich's (2016:1) initial questions in The Science of Culture? Social Computing, Digital Humanities and Cultural Analytics, are considered in the project:

What are the unique possibilities offered by computational analysis of large cultural data in contrast to qualitative methods used in humanities and social science? How can we combine computational analysis and visualization of large cultural data with qualitative methods (...)?

In other words, how does one combine analysis of larger patterns with the analysis of individual artifacts and their details?

IDEAS is being developed to enable the study of a considerably large number of cases. The data is going to be gathered in an open access web platform as an Architecture Wiki. In other words, IDEAS is a public global archive of transdisciplinary spatial practices, architecture production, research projects, theory, technologies that are being harnessed I creative research practices that are concerned with current cultural and ecological challenges.

When online, IDEAS is going to be a digital interactive interface to discover experimental practices and explore how the projects were developed. The database creation is following the process of scoping, observing, examining, and classifying, based in 3 deliverables: (1) design a timeline; (2) create a taxonomy classification and analysis; and (3) create the web-based database IDEAS.

These stages sync with the most important operators of archival practices: recording, coding, structuring, storing, processing, making accessible, copying/multiplying (Szekely, 2017). 


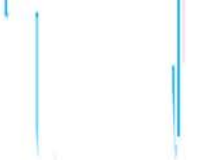

TECHNOCULURE CULTURAL LANDSCAPE IIMELINE

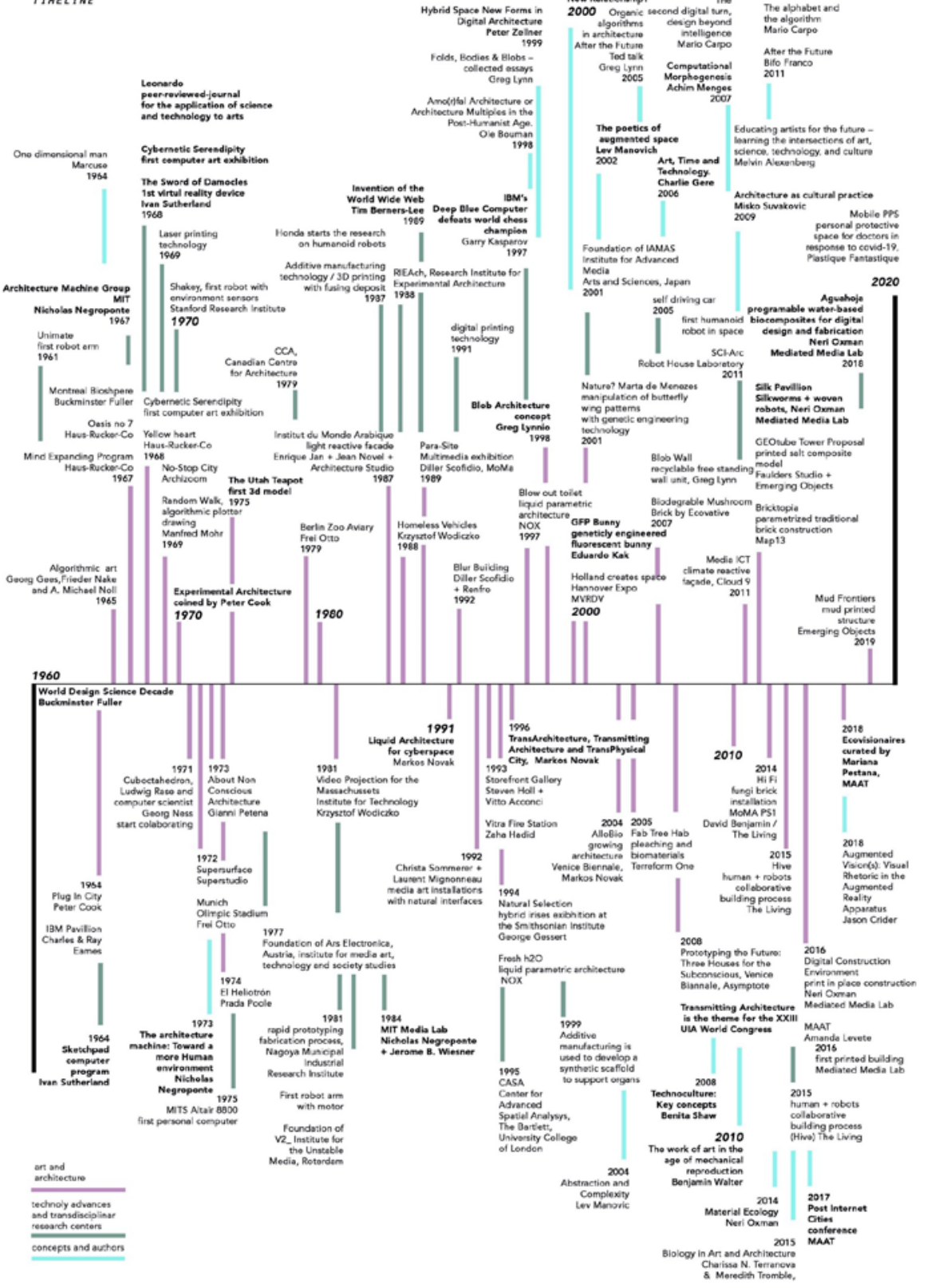

Figure 1. Prototype of the technoculture and experimental architecture timeline. Source: Raquel Lopes, 2020. 


\subsection{Stage 2: examining and classifying}

In this stage, we will process the information that was gathered during the timeline conception using open access qualitative data analysis software (QDA Miner Lite) to process, analyse and catalogue the retrieved data. The processing will include the classification of the different selected works, the inclusion of data sets, and metadata and features for each project that is being analysed.

Due to the large chosen analysis period (1960-2020), the file formats will be considerably heterogeneous. Note that we will be gathering hand made sketches, collages, models, letters, texts, catalogues, specifications notes, contracts, and photographs from the 1960-1980 period. In the digital period, 1980-2020, when digital technologies were more commonly used in spatial practices, we will be considering CAD files, spreadsheets, video, 3D models, code, plug-ins, and Virtual Reality.

QDA Miner Lite enables the processing of various file formats, its organization in an embedded exhaustive cataloguing system, as well as associating metadata and features in all entities. For all the mentioned reasons, it is a powerful key tool to develop our project.

This stage will enable the creation of an interactive taxonomy that will be the base for IDEAS. We intend to identify, catalogue and cluster experimental spatial practices to expose the process that enabled its development and implementation. In table 1, we show our proposal of data sets for the 4 branches of cultural production we intend to process and analyse. Note that, despite sharing some of the main features, not all branches can be characterised by all the data sets.

In a zoomed-out point of view, this will enable the identification, and classification of emerging research fields within spatial production and building, harnessed interdisciplinary and transdisciplinary methodologies and new spatial programs.

\begin{tabular}{|c|c|c|c|c|c|}
\hline Main Data Sets & \multirow[t]{2}{*}{ SPACE } & \multirow[t]{2}{*}{ TIME } & \multirow[t]{2}{*}{ FUNCTION } & \multirow[t]{2}{*}{ CONCEPTION } & \multirow[t]{2}{*}{ METHODOLOGY } \\
\hline $\begin{array}{l}\text { Embebded } \\
\text { metadata }\end{array}$ & & & & & \\
\hline $\begin{array}{l}\text { Art and } \\
\text { Architecture }\end{array}$ & 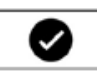 & $\theta$ & $\theta$ & 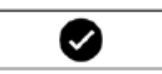 & $\theta$ \\
\hline $\begin{array}{l}\text { Transdisciplinary } \\
\text { Research } \\
\text { Centers }\end{array}$ & $\checkmark$ & $x$ & $\varnothing$ & 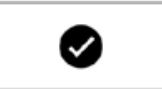 & 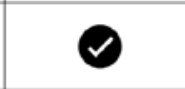 \\
\hline $\begin{array}{l}\text { Technology } \\
\text { Developments }\end{array}$ & 2 & (x) & 2 & $\varnothing$ & 2 \\
\hline $\begin{array}{l}\text { Concepts and } \\
\text { Author }\end{array}$ & $\otimes$ & (x) & V & 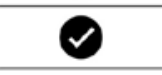 & $\times$ \\
\hline
\end{tabular}

Table 1. Taxonomy chart with the first layers of datasets Source: Raquel Lopes, 2021 


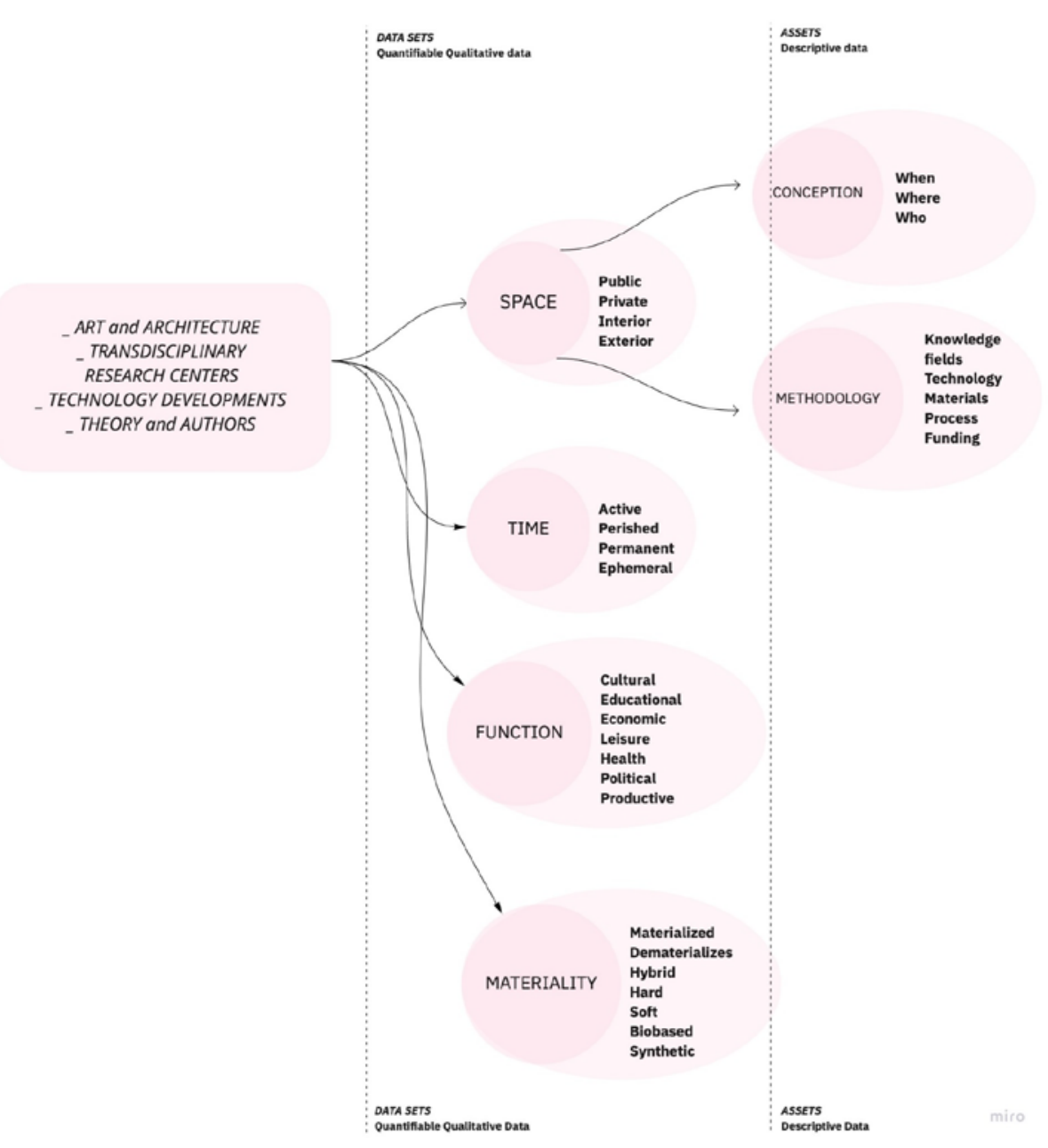

Figure 2. Taxonomy map

Source: Raquel Lopes, 2021

\subsection{Stage 3: website development}

The Interactive Data Base of Experimental Architecture and Spatial Practices (IDEAS) contributes to promote transdisciplinarity and plurality in spatial practices. The platform will make retrieving accessible while combining data sets to help users exploring experimental spatial practices. IDEAS will be an open access platform hosted in ISTAR-IUL web server and developed with the 
help of ISTAR-IUL programmers, featuring the research work developed during stage 1 and 2 of the project (timeline and taxonomy).

IDEAS will be a multi-layer system, navigable with the association of different keywords that are correspondent to data sets. The projects will be clustered, organized and associated when sharing data sets and metadata, according to, for example, knowledge fields, type of project, used materials and technologies. One will be able to access IDEAS and choose (associating) provided keywords to search all projects that share the same data sets.

The search engine will provide two different visualization options for all the projects that share the selected keywords: organized by colour coded georeferencing or colour coded data clustering.

Color coded geo-referencing will map each project in a representation of Earth. We believe geo-referenciacing will offer interesting analysis opportunities, such as understanding if there are regional tendencies, if the existence of research centers and universities has a direct impact in spatial practices, what are the matters that are being more explored and researched in different locations, for example. The schemes will be colour coded to enable an easy understanding of the data visualization, and immediately recognise the 4 branches of analysis the database provides: art and architecture production; transdisciplinary research centres; digital technology development; concepts and authors.

The clustering view will group the entrances in clusters of projects that share data sets. The distance between them will differ in accordance with the number of data sets they share, creating a network of similarities.

In both views, the users can choose and click on each project to enter in a multi layered information system.

We intend to develop dendrograms to provide a visual and layered taxonomy of each entry, allowing the users to choose the level of information they want to access and retrieve. The application of granularity will allow the analysis of spatial practices in their micro and macro scales, cataloguing, pinpointing and detailing projects, while connecting them with others with shared data sets.

The project's dendrogram's first layers will correspond to the data sets Time, Space, What, When, Where, displayed in table 1 . This will create an easy identification system. to briefly describe each project.

In deeper layers of the taxonomy, the metadata and features will be, in some cases, highly technical. These will correspond to the data sets Who and How. The metadata will inform how each project was developed, which methodologies were applied, what kind of technologies were used. We consider this an interesting aspect of IDEAS, allowing the user to explore the level of information, detail, and technicality they want to retrieve from each project.

In our opinion, the database can be a powerful tool for students, artists, theorists, researchers, space practitioners, public policy offices, as well as curious public. We expect it to be an inspiring platform, to help practitioners expand their scope of action and embed transdisciplinary practices and different knowledge fields in their practice. We see architecture and spatial 
practices as a technology to solve local and specific challenges, and hybridization to reach spatial plurality and creative meaningful urban territories

\section{Preliminary Results}

Despite being in the first stages of development, the project is already presenting the first challenges and topics for reflection. Regarding this, we see it is important to keep the framework flexible and adjust the different stages methodology to the challenges we are facing now and will face in the stages to come in the near future.

The first challenge we must overcome is the timeline creation, as it is a crucial stage of the project.

In this case, the number of entries doesn't correlate directly with the quality of the database and taxonomy we intend to create. The creation of a saturated timeline is important to develop a critical theory for transdisciplinary and experimental practices, but it is also crucial to select cases that can provide us all the information we want to embed and allow us to show and explain the processes that led to final project.

In this case, the process of scoping and mapping is a laborious stage that must be done establishing connections with the architects, research centers, museums, curators, scientists that were part of the projects, and guarantee that they can share information, data, documents, to enable us to explain and show how the projects were developed.

IDEAS is a platform to share knowledge and methodologies and relies on the same aspect: authors need to share their work with us. Most probably, this will help narrow the scope of entries and projects that will be featured in the deeper layers of IDEAS.

Another aspect and challenge of the process is the data set creation for experimental and transdisciplinary projects and how to create homogeneity within plurality.

Besides the main data sets presented in Table 1-Time, Space, What, When, Where, Who, How, we will develop a secondary layer of data sets. This layer will be specific for each one of the 4 branches of cultural production we are analysing (Art and Architecture, Transdisciplinary Research Centers, Technology Developments, and Concepts and Authors).

The main connections between the 4 branches will be established within the first layer of data sets.

The second layer of data sets will enable a stronger and more detailed connection between the projects that are showcased in each specific branch. This secondary layer will be a deconstruction of the How data set.

The embedded metadata will provide an under layer of information, enabling the visualization of relations between works in the different branches we are analysing. 


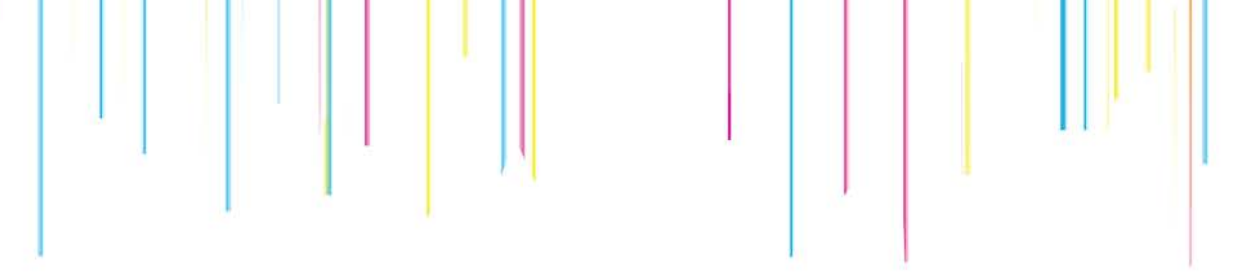

\section{Discussion and Conclusions}

IDEAS enables the development of a critical review of experimental architecture practices and research, having in consideration advances in technology that changed the state-of-the-art. Most importantly, the platform intends to inspire practitioners to expand their scope of action within their practice.

IDEAS will be a functional tool, helping to understand o spatial projects can be transformed into richer opportunities and how to create a bigger impact through transdisciplinarity. As Lev Manovic (2016:14) states in The Science of Culture? Social Computing, Digital Humanities and Cultural Analytics, the goal of such "wide data analysis" will be not only to find new similarities, affinities, and clusters in the universe of cultural artifacts, but, first of all, to help us question our commonsense view of things.

IDEAS and its different stages can migrate from digital to analogical modes of dissemination. IDEAS timeline, taxonomies and archive can be presented in exhibition spaces as well as creating curatorial programs around the topics of transdisciplinarity, experimental spatial practices and the impact of technology in cultural production.

As researchers, it is also critical to study how IDEAS can become an open and collaborative project, such as Wikipedia, and how to guarantee the quality and quantity of metadata. Currently, we believe this can be achieved by applying a pre-established data set system and programming the platform to only accept new entrances if the upload can fill the established data sets, and fulfills a certain quantity of embedded metadata, besides verify image quality and size.

\section{Aknowledgments}

This work is partially funded by national funds through FCT - Fundação para a Ciência e Tecnologia, I.P., under the project FCT UIDB/04466/2020 


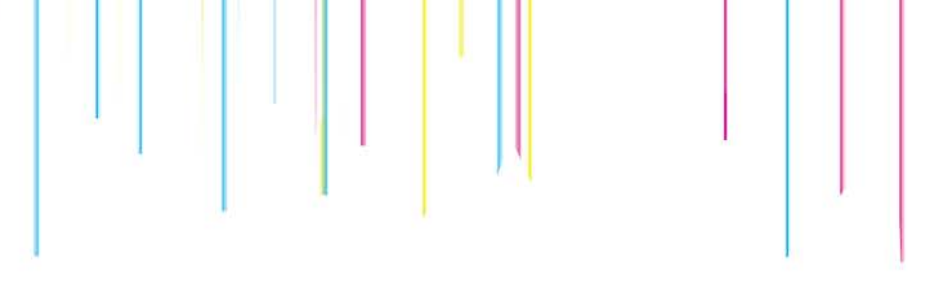

\section{References}

Bernstein, J. (2015). Transdisciplinarity: A Review of its Origins, Development and Current Issues. Journal of Research Practice, Volume 11(1), Article 1.

Fuller, B; McHalle, J. (1963). Inventory of the World Resources, Human Trends and Needs. https://www.bfi.org/sites/default/files/attachments/literature_source/wdsd_ phase1_doc1_inventory.pdf

Burry, M. \& Cutler, T. (2009). Designing solutions to wicked problems: $A$ manifesto for transdisciplinary research and design. RMIT Design Research Institute.

Carpo, M. (2017). The Second Digital Turn, Design Beyond Intelligence. The MIT Press.

Carpo, M. (2018). Big data and the end of history. International Journal for Digital Art History, 3 (July), 21-35. https://doi.org/10.11588/dah.2018.3.49913

Cook, P. (1970). Experimental Architecture. Universe Books.

Cooper, S. (2002). Technoculture and Critical Theory, In Service of the Machine? Routledge.

Dave, B. (2005). Digital Speculations. Leonardo Electronic Almanac 13(6/7), 14.

Dodd, M. 2020. Spatial Practices. Modes of Action and Engagement with the City. Routledge.

Doucet, I. \& Janssens, N. (2011). Transdisciplinary Knowledge Production in Architecture and Urbanism: Towards Hybrid Modes of Inquiry. Springer.

Gere, C. (2006). Art, Time and Technology. Berg.

Jencks, C. (1971). Architecture 2000: Predictions and Methods (new concepts of architecture). Praeger.

Jencks, C. (2000). Architecture 2000 and Beyond: success in the art of prediction. Wiley.

Kak, E. (2007). Signs of life: Bio art and beyond. The MIT Press.

Koolhaas, R. (2019, January-February). Museum in the Countryside, Aesthetics of the Data Center. IN N. Young (Ed.) Machine Landscapes, Architectures of the Post Anthropocene (pp. 60-65). Architectural Design.

Leavy, P. (2017). Research design. Guilford Press. 


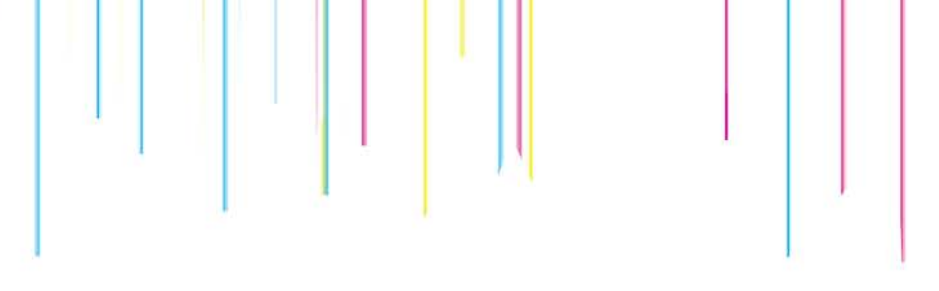

Lefebvre, H. (1974). The Production of Space. Wiley-Blackwell.

Leitão, C.(2012, April 16). Cloud Architecture. The Huffington Post. https://www.huffpost.com/entry/cloudarchitecture_b_848018\#s268693\&title=Self_Repairing_Architecture

Leopold, C., Robeller, C.\& Weber, U. (2020). Research Culture in Architecture. CrossDisciplinary Collaboration. Birkhäuser.

Ligget, H. \& Perry, D. (1995). Spatial Practices - Critical Explorations in Social/Spatial Theory. Sage Publications.

Maldonado, T. (2012). Cultura, Sociedade e Técnica. Blucher.

Manovic, L. (2016). The Science of Culture? Social Computing, Digital Humanities and Cultural Analytics. Journal of Cultural AnaliticsAnalytics Volume 1(1), 1-15. doi: 10.22148/16.00

Melendez, F., Diniz, N. \& Signore, M. (2021). Data, Matter, Design. Strategies in Computational Design. Routledge.

Mueller, A. (2009). Dutch Artistic Research Event \#3. Mahkuzine Journal of Artistic Research, 6, 5-8.

Shaw, B. (2008). Technoculture: Key Concepts. Berg.

Signore, M. \& Riether, G. (2020). Urban Machines: Public Space in Digital Culture. LISTLAB.

Stalder, F. (2018). The Digital Condition. Wiley.

Stokols, D. (2006). Toward a Science of Transdisciplinary Action Research. American Journal of Community Psychology 38, 63-77. https://doi.org/10.1007/s10464-0069060-5

Szekely, I. (2017). Do Archives have a Future in the Digital Age? Journal of Contemporary Archival Studies Volume 4 (2),1-16.

Vidler, A. (2004). Architecture's Expanded field. Artforum 42(8), 142-147.

Wilson, S. (2007). Beyond the digital: Preparing Artists to Work at the Frontiers of Techno-Culture. IN M. Alexenberg (Ed.) Learning at the Intersections of Art, Science, Technology, and Culture (pp. 29-45). Intelect Books.

Woiseth, H. \& Nilsson, F. (2011). Building (Trans)Disciplinary Architecture Research Introducing Mode 1 and mode 2 to Design Practitioners. IN I. Doucet \& N. Janssens (Eds.) Transdisciplinary Knowledge Production in Architecture and Urbanism. Urban and Landscape Perspectives (pp. 79-96). Springer, https://doi.org/10.1007/978-94-007-0104-5_6

Young, L. (2019). Neo Machine, Architecture Without People. IN N. Young (Ed.) Machine Landscapes, Architectures of the Post Anthropocene (pp. 6-13). Architectural Design.

Zellner, P. (1999) Hybrid Space: New forms in Digital Architecture. Rizzzoli International Publications. 